\title{
The (G'/G)-Expansion Method for Traveling Wave Solutions of Burgers' Kdv and Generalization of Huxley Equations
}

\author{
Rajan Arora, Sanjay Yadav* \\ Indian Institute of Technology, Roorkee, Saharanpur Campus, Saharanpur U.P., 247001, India
}

\begin{abstract}
The (G`/G)-expansion method is used for determining the exact traveling wave solutions of the Burgers-KdV and generalization of Huxley equations. The obtained solutions are compared with the solutions found by Wazwaz[18]. The $\left(\mathrm{G}^{`} / \mathrm{G}\right)$-method is very powerful and easy tool for solving non-linear partial differential equations
\end{abstract}

Keywords $\left(G^{\prime} / G\right)$-expansion method, Burgers KDV equation, generalization of Huxley equation, traveling wave solutions, exact solution

\section{Introduction}

Nonlinear equations in mathematical physics appear in various areas, such as fluid dynamics, plasma physics, optical fibers, solid state physics and other applications. A variety of powerful methods have been used to study the nonlinear equations such as the homotopy perturbation $\operatorname{method}[1]$, the variational iteration method[2], Hirotas bilinear methods[3], the sine-cosine function method[4-5], Jacobi elliptic method[6], the standard tanh and extended tanh methods[7-11], the exp-function method[12-13], the inverse scattering method[14] and so on. One of the most powerful and direct methods for constructing solutions of non-linear equations is the $\left(\mathrm{G}^{`} / \mathrm{G}\right)$-expansion method $[15$ 17]. This method was first introduced by Wang et al.[15] and it has been widely used for finding various exact solutions of non-linear partial differential equations. The computer symbolic systems such as Maple and Mathematica allow us to perform complicated and tedious calculations The parameter $m$ plays an important role in the $\left(\mathrm{G}^{\prime} / \mathrm{G}\right)$ expansion method; it should be a positive integer to derive a closed form analytic solution. However, for non-integer values of $m$, we usually apply a transformation formula to overcome this difficulty.

We begin our study by examining the Burgers-KdV and generalization of Huxley equation [18]

$$
\begin{aligned}
& u_{t}+a\left(u^{n}\right)_{x}-b u_{x x}+u_{x x}=0, \quad n>1, \quad a, b \neq 0 \\
& u_{t}-a u_{x x}-u\left(k-u^{n}\right)\left(u^{n}-1\right)=0, \quad n>1, \quad a \neq 0
\end{aligned}
$$

* Corresponding author:

Sanjayyadav.yadav91@gmail.com (Sanjay Yadav)

Published online at http://journal.sapub.org/ajcam

Copyright (C) 2012 Scientific \& Academic Publishing. All Rights Reserved where the dependent variable $u$ is the function of space variable $x$ and time variable $t ; a, b$ are the non-zero arbitrary constants. Finding exact traveling wave solutions for the above non-linear partial differential equations, by using $\left(\mathrm{G}^{`} / \mathrm{G}\right)$-expansion method, is our goal here.

\section{Analysis of the (G`/G)-expansion method}

The $\left(\mathrm{G}^{\prime} / \mathrm{G}\right)$-expansion method $([15-17])$ is a powerful solution method for the computation of exact traveling wave solutions of partial differential equations (PDEs).

We consider non-linear PDE for $u(x, t)$ in the form:

$$
P\left(u, u_{t}, u_{x}, u_{x x}, u_{t t}, u_{x t}, \ldots\right)=0 \text {, }
$$

where $u(x, t)$ is the unknown function depending on space variable $x$ and time variable $t, P$ is a polynomial in $u(x, t)$ and its partial derivatives, in which the highest order derivatives and nonlinear terms are involved. To obtain the traveling wave solution of PDE (2), we introduce the variable $\xi=x-\omega t$ so that $u(x, t)=U(\xi)$, where $\omega$ is a constant. We use the following change of partial derivatives

$$
\frac{\partial}{\partial t}=-\omega \frac{d}{d \xi}, \quad \frac{\partial}{\partial x}=\frac{d}{d \xi}, \quad \frac{\partial^{2}}{\partial x^{2}}=\frac{d^{2}}{d \xi^{2}},
$$

and so on for the other derivatives. Thus PDE (2) reduces to an ordinary differential equation (ODE)

$$
Q\left(U, U^{\prime}, U^{\prime \prime}, U^{\prime \prime \prime}, \ldots\right)=0 \text {, }
$$

where the primes denote the derivative with respect to $\xi$. Equation (3) is then integrated as long as all the terms contain derivatives, where integration constants are considered to be zero.

Now, we assume that the solution of the ODE (3) can be expressed by a polynomial in $\left(\mathrm{G}^{\prime} / \mathrm{G}\right)$ as follows: 


$$
U=\sum_{i=1}^{m} \alpha_{i}\left(\frac{G^{\prime}}{G}\right)^{i}+\alpha_{0}, \quad \alpha_{m} \neq 0,
$$

where $G=G(\xi)$ satisfies the second order linear ODE in the form

$$
G^{\prime \prime}+\lambda G^{\prime}+\mu G=0
$$

where $G^{\prime}=\frac{d G}{d \xi}, G^{\prime \prime}=\frac{d^{2} G}{d \xi^{2}}$, and $\alpha_{m} \neq 0$,

$\alpha_{1}, \alpha_{0}, \lambda$ and $\mu$ are real constants which are to be determined.

Using (4) and (5), we obtain

$$
\begin{gathered}
U^{\prime}=-\sum_{i=1}^{m} i \alpha_{i}\left[\left(\frac{G^{\prime}}{G}\right)^{i+1}+\lambda\left(\frac{G^{\prime}}{G}\right)^{i}+\mu\left(\frac{G^{\prime}}{G}\right)^{i-1}\right], \\
U^{\prime \prime}=\sum_{i=1}^{m} i \alpha_{i}\left[\begin{array}{l}
(i+1)\left(\frac{G^{\prime}}{G}\right)^{i+2}+(2 i+1) \lambda\left(\frac{G^{\prime}}{G}\right)^{i+1} \\
+i\left(\lambda^{2}+2 \mu\right)\left(\frac{G^{\prime}}{G}\right)^{i}+(2 i-1) \lambda \mu\left(\frac{G^{\prime}}{G}\right)^{i-1} \\
+(i-1) \mu^{2}\left(\frac{G}{G}\right)^{i-2}
\end{array}\right]
\end{gathered}
$$

Using the general solution of (5), we have for $\lambda^{2}-4 \mu>0$,

$$
\left(\frac{G^{\prime}}{G}\right)=\frac{\sqrt{\lambda^{2}-4 \mu}}{2}\left(\frac{c_{1} \sinh \frac{1}{2} \sqrt{\lambda^{2}-4 \mu} \xi+c_{2} \cosh \frac{1}{2} \sqrt{\lambda^{2}-4 \mu} \xi}{c_{1} \cosh \frac{1}{2} \sqrt{\lambda^{2}-4 \mu} \xi+c_{2} \sinh \frac{1}{2} \sqrt{\lambda^{2}-4 \mu} \xi}\right)-\frac{\lambda}{2},
$$

for $\lambda^{2}-4 \mu<0$,

$$
\left(\frac{G^{\prime}}{G}\right)=\frac{\sqrt{4 \mu-\lambda^{2}}}{2}\left(\frac{-c_{1} \sin \frac{1}{2} \sqrt{4 \mu-\lambda^{2}} \xi+c_{2} \cos \frac{1}{2} \sqrt{4 \mu-\lambda^{2}} \xi}{c_{1} \cos \frac{1}{2} \sqrt{4 \mu-\lambda^{2}} \xi+c_{2} \sin \frac{1}{2} \sqrt{4 \mu-\lambda^{2}} \xi}\right)-\frac{\lambda}{2} .
$$

Also for $\lambda^{2}-4 \mu=0$,

$$
\left(G^{\prime} / G\right)=-\lambda / 2 \text {. }
$$

To determine $\mathrm{U}$ explicitly, we take the following four steps:

Step 1. Determine the integer $m$ by substituting (4) along with (5) into (3), and balancing the highest order nonlinear term(s) and the highest order partial derivative.

Step 2. By substituting (4) and (5) into (3) with the value of $m$ obtained in Step 1, and collecting all term(s) with the same order of $\left(G^{\prime} / G\right)$ together, the left-hand side of (3) converts into polynomial in $\left(G^{\prime} / G\right)$. Then setting the coefficients of $\left(G^{\prime} / G\right)^{i}(i=0,1,2)$ to zero, we obtain a set of algebraic equations in $\alpha_{1}, \alpha_{0}, \omega, \lambda$ and $\mu$.

Step 3. Solve the system of algebraic equations obtained in step 2 for $\alpha_{1}, \alpha_{0}$, and $\mu$ by use of Mathematica.

Step 4. By substituting the results obtained in the above steps, we can obtain exact traveling wave solutions of (2).

\section{Applications}

In this section, we apply the $\left(\mathrm{G}^{`} / \mathrm{G}\right)$-expansion method to construct the traveling wave solution of Burgers-KdV and generalization of Huxley equations.

\subsection{The Burgers-Kdv equation}

The Burgers-Kdv equation is given by

$$
u_{t}+a\left(u^{n}\right)_{x}-b u_{x x x}+u_{x x}=0, n
$$

Using the transformation $\mathrm{u}(\mathrm{x}, \mathrm{t})=\mathrm{U}(\xi)$, where $\xi=x-\omega t$, the PDE is reduced to an ODE

$$
-\omega U^{\prime}+a\left(U^{n}\right)^{\prime}-b\left(U^{\prime \prime \prime}\right)+U^{\prime \prime}=0,
$$

where the primes denote the derivative with respect to $\xi$. Integrating once with respect to $\xi$, and taking constant of integration to be zero, (11) reduces to

$$
-\omega U+a\left(U^{n}\right)-b\left(U^{\prime \prime}\right)+U^{\prime}=0 .
$$

Balancing $U^{n}$ and $U^{n}$,

$$
M=\frac{2}{n-1}, \quad n>1 \text {. }
$$

Using the transformation

$$
U=V^{\frac{2}{n-1}},
$$

equation (12) converts to

$$
\begin{aligned}
& -\omega(n-1)^{2} V^{2}+a(n-1)^{2} V^{4}-2 b(n-1) V V^{\prime \prime} \\
& -2 b(3-n)\left(V^{\prime}\right)^{2}+2(n-1) V V^{\prime}=0 .
\end{aligned}
$$

Now balancing $V V^{n}$ with $V^{4}$ i.e. $m+m+2=4 m$, we obtain $m=1$. Therefore, we assume the solution of (15) in the form

$$
V=\alpha_{0}+\alpha_{1}\left(\frac{G^{\prime}}{G}\right), \quad \alpha_{1} \neq 0 .
$$

Using (6), (7) and (16), we obtain

$$
\begin{aligned}
V^{\prime}= & -\alpha_{1}\left(\frac{G^{\prime}}{G}\right)^{2}-\alpha_{1} \lambda\left(\frac{G^{\prime}}{G}\right)-\alpha_{1} \mu, \\
V^{4}= & \alpha_{0}^{4}+\alpha_{1}^{4}\left(\frac{G^{\prime}}{G}\right)^{4}+6 \alpha_{0}^{2} \alpha_{1}^{2}\left(\frac{G^{\prime}}{G}\right)^{2} \\
& +4 \alpha_{0}^{3} \alpha_{1}\left(\frac{G^{\prime}}{G}\right)+4 \alpha_{0} \alpha_{1}^{3}\left(\frac{G^{\prime}}{G}\right)^{3}, \\
\left(V^{\prime}\right)^{2}= & \alpha_{1}^{2}\left(\frac{G^{\prime}}{G}\right)^{4}+\alpha_{1}^{2} \lambda^{2}\left(\frac{G^{\prime}}{G}\right)^{2}+\alpha_{1}^{2} \mu^{2} \\
& +2 \alpha_{1}^{2} \lambda\left(\frac{G^{\prime}}{G}\right)^{3}+2 \alpha_{1}^{2} \mu\left(\frac{G^{\prime}}{G}\right)^{2}+2 \alpha_{1}^{2} \lambda \mu\left(\frac{G^{\prime}}{G}\right), \\
V^{\prime \prime}= & 2 \alpha_{1}\left(\frac{G^{\prime}}{G}\right)^{3}+3 \alpha_{1} \lambda\left(\frac{G^{\prime}}{G}\right)^{2} \\
& +\left(\alpha_{1} \lambda^{2}+2 \alpha_{1} \mu\right)\left(\frac{G^{\prime}}{G}\right)^{2}+\alpha_{1} \lambda \mu .
\end{aligned}
$$

Now using (16)-(20) in (15) and equating the coefficients of $\left(\frac{G^{\prime}}{G}\right)^{i},(i=0,1,2,3,4)$ to zero, we obtain a system of algebraic equations in $\alpha_{1}, \alpha_{0}, \omega, \lambda$ and $\mu$ as follows:

$$
\begin{aligned}
\left(\frac{G^{\prime}}{G}\right)^{0}: & -\omega(n-1)^{2} \alpha_{0}^{2}+a(n-1)^{2} \alpha_{0}^{4}-2 b(n-1) \alpha_{0} \alpha_{1} \lambda \mu \\
& -2 b(3-n) \alpha_{1}^{2} \mu^{2}-2(n-1) \alpha_{0} \alpha_{1} \mu=0, \\
\left(\frac{G^{\prime}}{G}\right)^{1}: & -2 \omega(n-1)^{2} \alpha_{0} \alpha_{1}+4 a(n-1)^{2} \alpha_{0}^{3} \alpha_{1} \\
& -2 b(n-1)\left[\alpha_{0}\left(\alpha_{1} \lambda^{2}+2 \alpha_{1} \mu\right)+\alpha_{1}^{2} \lambda \mu\right] \\
& -4 b(3-n) \alpha_{1}^{2} \lambda \mu+2(n-1)\left[-\alpha_{0} \alpha_{1} \lambda-\alpha_{1}^{2} \mu\right]=0,
\end{aligned}
$$




$$
\begin{aligned}
&\left(\frac{G^{\prime}}{G}\right)^{2}:-\omega(n-1)^{2} \alpha_{1}^{2}+6 a \alpha_{0}^{2} \alpha_{1}^{2}(n-1)^{2} \\
&-2 b(n-1)\left[3 \alpha_{0} \alpha_{1} \lambda+\alpha_{1}\left(\alpha_{1} \lambda^{2}+2 \alpha_{1} \mu\right)\right] \\
&-2 b(3-n)\left[\alpha_{1}^{2} \lambda^{2}+2 \alpha_{1}^{2} \mu\right]+2(n-1)\left[-\alpha_{0} \alpha_{1}-\alpha_{1}^{2} \lambda\right]=0, \\
&\left(\frac{G^{\prime}}{G}\right)^{3}: 4 a(n-1)^{2} \alpha_{0} \alpha_{1}^{3}-2 b(n-1)\left[2 \alpha_{0} \alpha_{1}+3 \alpha_{1}^{2} \lambda\right] \\
& \quad-4 b(3-n) \alpha_{1}^{2} \lambda-2(n-1) \alpha_{1}^{2}=0 \\
&\left(\frac{G^{\prime}}{G}\right)^{4}: a(n-1)^{2} \alpha_{1}^{4}-4 b \alpha_{1}^{2}(n-1)-2 b \alpha_{1}^{2}(3-n)=0 .
\end{aligned}
$$

Solving the system of equations (21)-(25) by using MATHEMATICA, we obtain the following two sets of solutions for $n=2$ :

Set1: $\omega=\frac{6}{25 b}, \quad \mu=\frac{-1+25 b^{2} \lambda^{2}}{100 b^{2}}$,

$\quad \alpha_{1}=-\frac{\sqrt{6 b}}{\sqrt{a}}, \quad \alpha_{0}=-\frac{\sqrt{6}(1+5 b \lambda)}{10 \sqrt{a b}}$.

Set2: $\omega=\frac{6}{25 b}, \quad \mu=\frac{-1+25 b^{2} \lambda^{2}}{100 b^{2}}$,

$$
\alpha_{1}=\frac{\sqrt{6 b}}{\sqrt{a}}, \quad \alpha_{0}=\frac{\sqrt{6}(1+5 b \lambda)}{10 \sqrt{a b}} .
$$

Hence, for Set 1:

$$
V(\xi)=-\frac{\sqrt{6}(1+5 b \lambda)}{10 \sqrt{a b}}-\frac{\sqrt{6 b}}{\sqrt{a}}\left(\frac{G^{\prime}}{G}\right) .
$$

Now, using $U=V^{\frac{2}{n-1}}=V^{2}$,

$$
U(\xi)=\left\{-\frac{\sqrt{6}(1+5 b \lambda)}{10 \sqrt{a b}}-\frac{\sqrt{6 b}}{\sqrt{a}}\left(\frac{G^{\prime}}{G}\right)\right\}^{2} .
$$

When $\lambda^{2}-4 \mu>0$, we obtain hyperbolic function solution of the Burgers-KdV equation (10) as

$$
U(\xi)=\left[\begin{array}{l}
-\frac{\sqrt{6}(1+5 b \lambda)}{10 \sqrt{a b}}-\frac{\sqrt{6 b}}{\sqrt{a}} \times \\
\left\{\frac{\sqrt{\lambda^{2}-4 \mu}}{2}\left(\frac{C_{1} \sinh \frac{1}{2} \sqrt{\lambda^{2}-4 \mu} \xi+C_{2} \cosh \frac{1}{2} \sqrt{\lambda^{2}-4 \mu} \xi}{C_{1} \cosh \frac{1}{2} \sqrt{\lambda^{2}-4 \mu} \xi+C_{2} \sinh \frac{1}{2} \sqrt{\lambda^{2}-4 \mu} \xi}\right)-\frac{\lambda}{2}\right\}
\end{array}\right]^{2},
$$

when $\lambda^{2}-4 \mu<0$, we obtain the trigonometric function solution of the Burgers-KdV equation (10) as

$$
U(\xi)=\left[\begin{array}{l}
-\frac{\sqrt{6}(1+5 b \lambda)}{10 \sqrt{a b}}-\frac{\sqrt{6 b}}{\sqrt{a}} \times \\
\left\{\frac{\sqrt{4 \mu-\lambda^{2}}}{2}\left(\frac{-C_{1} \sin \frac{1}{2} \sqrt{4 \mu-\lambda^{2}} \xi+C_{2} \cos \frac{1}{2} \sqrt{4 \mu-\lambda^{2}} \xi}{C_{1} \cos \frac{1}{2} \sqrt{4 \mu-\lambda^{2}} \xi+C_{2} \sin \frac{1}{2} \sqrt{4 \mu-\lambda^{2}} \xi}\right)-\frac{\lambda}{2}\right\}
\end{array}\right]^{2},
$$

and when $\lambda^{2}-4 \mu=0$,

$$
U(\xi)= \pm \frac{\sqrt{6}(1+5 b \lambda)}{10 \sqrt{a b}} \mp \frac{\sqrt{6 b} \lambda}{2 \sqrt{a}} \text {. }
$$

If we set $C_{1} \neq 0, C_{2}=0$ in (28), we obtain

$u(x, t)=\left[-\frac{\sqrt{6}}{10 \sqrt{a b}}\left\{1+\tanh \frac{1}{10 b}\left(x-\frac{6}{25 b} t\right)\right\}\right]^{2}$.

If we set $C_{1}=0, C_{2} \neq 0$ in (28), we obtain

$$
u(x, t)=\left[-\frac{\sqrt{6}}{10 \sqrt{a b}}\left\{1+\operatorname{coth} \frac{1}{10 b}\left(x-\frac{6}{25 b} t\right)\right\}\right]^{2} .
$$

Similarly for Set 2:

$$
u(x, t)=\left[\frac{\sqrt{6}}{10 \sqrt{a b}}\left\{1+\tanh \frac{1}{10 b}\left(x-\frac{6}{25 b} t\right)\right\}\right]^{2}
$$

for $C_{1} \neq 0, \quad C_{2}=0$, and

$$
u(x, t)=\left[\frac{\sqrt{6}}{10 \sqrt{a b}}\left\{1+\operatorname{coth} \frac{1}{10 b}\left(x-\frac{6}{25 b} t\right)\right\}\right]^{2} .
$$

for $C_{1}=0, \quad C_{2} \neq 0$

\subsection{Generalization of Huxley equation}

Consider a generalization of the Huxley equation

$u_{t}-a u_{x x}-u\left(k-u^{n}\right)\left(u^{n}-1\right)=0$,

Proceeding as earlier, equation (35) is converted to the ODE

$$
-\omega U^{\prime}-a U^{\prime \prime}-(k+1) U^{n+1}+k U+U^{2 n+1}=0 .
$$

Now, balancing $U^{2 n+1}$ and $U^{\prime \prime}$, we find

$$
M=\frac{1}{n} \text {. }
$$

Using the transformation $U=V^{\frac{1}{n}}$ in (36), we obtain

$$
\begin{aligned}
& -\omega n V V^{\prime}-a n V V^{\prime \prime}-a(1-n)\left(V^{\prime}\right)^{2} \\
& -(k+1) n^{2} V^{3}+n^{2} V^{4}+k n^{2} V^{2}=0 .
\end{aligned}
$$

Now again balancing $V^{4}$ with $V V^{\prime \prime}$ i.e. $m+m+2=$ $4 m$, we obtain $m=1$. Therefore, $V$ assumes the same form as in (16). Now, putting the different values of $V, V^{\prime}, V^{\prime \prime}$ etc. from (16)-(20) in (37) and setting the coefficients of $\left(\frac{G^{\prime}}{G}\right)^{i}(i=0,1,2,3,4)$ to zero we obtain a system of algebraic equations in $\alpha_{1}, \alpha_{0}, \omega, \lambda$ and $\mu$ as follows:

$$
\begin{gathered}
\left(\frac{G^{\prime}}{G}\right)^{0}: \\
:\left(k n \alpha_{0} \alpha_{1} \mu-a n \alpha_{0} \alpha_{1} \mu \lambda-a(1-n) \alpha_{1}^{2} \mu^{2}\right. \\
-(k+1) n^{2} \alpha_{0}^{3}+n^{2} \alpha_{0}^{4}+k n^{2} \alpha_{0}^{2}=0,
\end{gathered}
$$

$$
\begin{aligned}
\left(\frac{G^{\prime}}{G}\right)^{1}: & \omega n \alpha_{0} \alpha_{1} \lambda+\omega n \alpha_{1}^{2} \mu-a n \alpha_{0}\left(\alpha_{1} \lambda^{2}+2 \alpha_{1} \mu\right) \\
& -a n \alpha_{1}^{2} \lambda \mu-2 a(1-n) \alpha_{1}^{2} \lambda \mu-3(k+1) n^{2} \alpha_{0}^{2} \alpha_{1} \\
& +4 n^{2} \alpha_{0}^{3} \alpha_{1}+2 k n^{2} \alpha_{0} \alpha_{1}=0 \\
\left(\frac{G^{\prime}}{G}\right)^{2}: & \omega n \alpha_{0} \alpha_{1}+\omega n \alpha_{1}^{2} \lambda-3 a n \alpha_{0} \alpha_{1} \lambda \\
& -a n \alpha_{1}\left(\alpha_{1} \lambda^{2}+2 \alpha_{1} \mu\right)-a(1-n)\left[\alpha_{1}^{2} \lambda^{2}+2 \alpha_{1}^{2} \mu\right] \\
& -3(k+1) n^{2} \alpha_{0} \alpha_{1}^{2}+6 n^{2} \alpha_{0}^{2} \alpha_{1}^{2}+k n^{2} \alpha_{1}^{2}=0 \\
\left(\frac{G^{\prime}}{G}\right)^{3}: & \omega n \alpha_{1}^{2}-2 a n \alpha_{0} \alpha_{1}-3 a n \alpha_{1}^{2} \lambda \\
& -2 a(1-n) \alpha_{1}^{2} \lambda-(k+1) n^{2} \alpha_{1}^{3}+4 n^{2} \alpha_{0} \alpha_{1}^{3}=0
\end{aligned}
$$




$$
\left(\frac{G^{\prime}}{G}\right)^{4}:-2 a n \alpha_{1}^{2}-a(1-n) \alpha_{1}^{2}+n^{2} \alpha_{1}^{4}=0 .
$$

Fixing $k=1, n=2$ and $a=1$, and then solving the system of equations (38)-(42) by MATHEMATICA, we obtain two sets of solutions:

Set 1:

$$
\omega=-\frac{2}{\sqrt{3}}, \quad \mu=\frac{-4+3 \lambda^{2}}{12}, \quad \alpha_{0}=\frac{2-\sqrt{3} \lambda}{4}, \quad \alpha_{1}=-\frac{\sqrt{3}}{2} .
$$

Set 2:

$$
\omega=\frac{2}{\sqrt{3}}, \quad \mu=\frac{-4+3 \lambda^{2}}{12}, \quad \alpha_{0}=\frac{2+\sqrt{3} \lambda}{4}, \quad \alpha_{1}=\frac{\sqrt{3}}{2} .
$$

Therefore, the solution of the generalization of the Huxley equation (35) corresponding to Set 1 using

$$
\begin{aligned}
U=V^{\frac{1}{n}}=V^{\frac{1}{2}}, \\
U(\xi)=\left[\frac{2-\sqrt{3} \lambda}{4}-\frac{\sqrt{3}}{2}\left(\frac{G^{\prime}}{G}\right)\right]^{\frac{1}{2}} .
\end{aligned}
$$

When $\lambda^{2}-4 \mu>0$,

$$
U(\xi)=\left[\begin{array}{l}
\frac{2-\sqrt{3} \lambda}{4}-\frac{\sqrt{3}}{2} \times \\
\left\{\frac{\sqrt{\lambda^{2}-4 \mu}}{2}\left(\frac{C_{1} \sinh \frac{1}{2} \sqrt{\lambda^{2}-4 \mu} \xi+C_{2} \cosh \frac{1}{2} \sqrt{\lambda^{2}-4 \mu} \xi}{C_{1} \cosh \frac{1}{2} \sqrt{\lambda^{2}-4 \mu} \xi+C_{2} \sinh \frac{1}{2} \sqrt{\lambda^{2}-4 \mu} \xi}\right)-\frac{\lambda}{2}\right)
\end{array}\right],
$$

when $\lambda^{2}-4 \mu<0$,

$$
U(\xi)=\left[\begin{array}{l}
\frac{2-\sqrt{3} \lambda}{4}-\frac{\sqrt{3}}{2} \times \\
\left\{\frac{\sqrt{4 \mu-\lambda^{2}}}{2}\left(\frac{-C_{1} \sin \frac{1}{2} \sqrt{4 \mu-\lambda^{2}} \xi+C_{2} \cos \frac{1}{2} \sqrt{4 \mu-\lambda^{2}} \xi}{C_{1} \cos \frac{1}{2} \sqrt{4 \mu-\lambda^{2}} \xi+C_{2} \sin \frac{1}{2} \sqrt{4 \mu-\lambda^{2}} \xi}\right)-\frac{\lambda}{2}\right\}
\end{array}\right],
$$

and when $\lambda^{2}-4 \mu=0$,

$$
U(\xi)=\frac{2 \pm \sqrt{3} \lambda}{4} \mp \frac{\sqrt{3} \lambda}{4} .
$$

If we set $C_{1} \neq 0, C_{2}=0$ in (44), we obtain

$$
u(x, t)=\left\{\frac{1}{2}\left(1-\tanh \frac{1}{\sqrt{3}}\left(x+\frac{2}{\sqrt{3}} t\right)\right)\right\}^{\frac{1}{2}} .
$$

If we set $C_{1}=0, C_{2} \neq 0$ in (44), we obtain

$$
u(x, t)=\left\{\frac{1}{2}\left(1-\operatorname{coth} \frac{1}{\sqrt{3}}\left(x+\frac{2}{\sqrt{3}} t\right)\right)\right\}^{\frac{1}{2}} .
$$

Similarly for Set 2

$$
u(x, t)=\left\{\frac{1}{2}\left(1+\tanh \frac{1}{\sqrt{3}}\left(x-\frac{2}{\sqrt{3}} t\right)\right)\right\}^{\frac{1}{2}}
$$

for $C_{1} \neq 0, \quad C_{2}=0$, and

$$
u(x, t)=\left\{\frac{1}{2}\left(1+\operatorname{coth} \frac{1}{\sqrt{3}}\left(x-\frac{2}{\sqrt{3}} t\right)\right)\right\}^{\frac{1}{2}},
$$

for $C_{1}=0, C_{2} \neq 0$.

\section{Conclusions}

$\left(\mathrm{G}^{\prime} / \mathrm{G}\right)$-expansion method is used to obtain the exact solutions of the Burgers-KdV and generalization of Huxley equations. The solution method is very simple and effective. The solutions are expressed in the form of hyperbolic functions and the trigonometric functions. It is shown that this method is a good tool for handling non-linear partial differential equations. The solutions are compared with the solutions obtained by Wazwaz[18], and it is found that the solutions obtained are exactly same as determined by Wazwaz[18].

\section{REFERENCES}

[1] He, J.H.: Application of Homotopy Perturbation Method to Nonlinear Wave Equations. Chaos, Solitons and Fractals 26, 695-700 (2005)

[2] He, J.H., Wu, X.H.: Construction of Solitary Solution and Compaction-Like Solution by Variational Iteration Method. Chaos Solitons and Fractals 29, 108-113 (2006)

[3] Hirota, R.: Exact Solution of the Kdv Equation for Multiple Collisions of Solitons. Physical Review Letters 27, 1192-1194 (1971)

[4] Borhanifar, A., Jafari, H., Karimi, S.A.: New solitary wave solutions for the bad Boussinesq and good Boussinesq equations, Numer. Methods for Partial Differential Equations 25, 1231-1237 (2009)

[5] Borhanifar, A., Jafari, H., Karimi, S.A.: New solitons and periodic solutions for the Kadomtsev-Petviashvili equation. Nonlinear Sci. Appl. 4, 224-229. (2008)

[6] Liu, G. T., Fan, T. Y.: New applications of developed Jacobi elliptic function expansion methods. Phys. Lett. A 345, 161-166 (2005)

[7] Wazwaz, A.M.: Exact solutions to the double sinh-Gordan equation by tanh method and a variable separated ode method. Compt. Math. Appl. 501685-1696 (2005)

[8] Malfliet, W., Hereman, W.: The tanh method: exact solutions of nonlinear evolution and wave equations. Phys. Scr. 54, 563-568 (1996)

[9] Wang, M.L.: Exact solution for a compound KdV-Burgers equations. Phys. Lett. A 213, 279-287 (1996)

[10] Malfliet, W.: Solitary wave solutions of nonlinear wave equations. Am. J. Phy. 60, 650-658 (1992)

[11] Malfliet, W., Hereman, W.: The tanh method: II. Perturbation technique for conservative systems. Phys Scr. 54, 569-575 (1996)

[12] Zhou, X.W., Wen, Y.X., He J.H.: Exp-function method to solve the nonlinear dispersive $\mathrm{k}(\mathrm{m}, \mathrm{n})$ equations, Int. J. Nonlinear Sci. Numer. Simul. 9, 301-306 (2008)

[13] Liu, G.T, Fan, T.Y.: New applications of developed Jacobi elliptic function expansion methods. Phys. Lett. A 345, 161-166 (2005) 
[14] Ablowitz, M.J., Segur, H.: Solitons and Inverse Scattering Transform. SIAM, Philadelphia (1981)

[15] Wang, M.L., Li, X. Zhang, J.: The (G'/G)-expansion method and traveling wave solutions of nonlinear evolution equations in mathematical physics. Phys. Lett. A 372 417-423 (2008)

[16] Aslan, İ.: Exact and explicit solutions to some nonlinear evolution equations by utilizing the $\left(\mathrm{G}^{\prime} / \mathrm{G}\right)$-expansion method.
Appl. Math. Comput. 215, 857-863 (2009)

[17] Bekir, A.: Application of the (G'/G)-expansion method for nonlinear evolution equations. Phys. Lett. A 372, 3400-3406 (2008)

[18] Wazwaz, A.M.: Travelling wave solutions of generalized forms of Burgers, Burgers-KDV and Burgers-Huxley equations. Appl. Math. Comput 169, 639-656 (2005) 